\title{
THE NEUROPHYSIOLOGICAL ACTIVATIONS OF NOVICE AND EXPERIENCED PROFESSIONALS WHEN DESIGNING AND PROBLEM-SOLVING
}

\author{
S. Vieira ${ }^{1, \otimes}$, J. Gero ${ }^{2}$, V. Gattol ${ }^{3}$, J. Delmoral ${ }^{4}$, S. Li ${ }^{1}$, G. Cascini ${ }^{1}$ and \\ A. Fernandes ${ }^{4}$ \\ ${ }^{1}$ Politecnico di Milano, Italy, ${ }^{2}$ UNC Charlotte, United States of America, ${ }^{3}$ AIT Austrian Institute of Technology, \\ Austria, ${ }^{4}$ University of Porto, Portugal \\ $\bigotimes$ sonia.dasilvavieira@polimi.it
}

\begin{abstract}
We present results from an EEG experiment EEG to measure neurophysiological activation to study novice and experienced designers when designing and problem-solving. We adopted and extended the tasks described in a previous fMRI study. The block experiment consists of 3 tasks: problem-solving, basic design, and open layout design. The block is preceded by a familiarizing pre-task and extended to an open design sketching task. Results from 36 sessions of mechanical engineers and industrial designers indicate significant differences in activations between the problem-solving and the design tasks.
\end{abstract}

Keywords: design science, problem solving, novices, experienced, design thinking

\section{Introduction}

Studies of the cognitive behavior of novice and experienced designers have been mainly based on methods from cognitive psychology, such as protocol analysis (Crutcher, 1994; Ericsson and Simon, 1984; Kan and Gero, 2017). The overlapping of the terms expert and experienced has populated design research as well. The term expert (Dreyfus and Dreyfus, 1988) is attributed to people that have an inherent know-how to do things, and as a stage of possible attainment for others. Expert's cognitive activity and productivity is almost three times as high as the novice's in terms of image generation (Kavakli and Gero, 2002). Their cognitive actions are well organized and clearly structured, while the novice's cognitive performance is divided into groups of concurrent actions (Kavakli and Gero, 2002). Experts and novices' performance differ in terms of their respective strategic knowledge. Experts are known for taking a systemic view of the design situation, framing the problem in a challenging way, and drawing upon first principles to guide the overall concept and detailed design (Cross and Clayburn Cross, 1998).

Results from the study of freshman and senior designers show that the latter produce higher quality solutions, spend more time solving the problem, consider alternative solutions and make more transitions between design steps than freshmen (Atman et al., 1999). The novice designers tended to use a particular pattern of trial and error. Experienced designers used particular design strategies. Experienced designers tend to combine between three to five activities before changing their approach to a particular problem and develop individual approaches to a design task. Individual approaches seem to be dependent on the type of design task (conceptual versus detail design) and also on age and experience (Ahmed et al., 2003). 
Experienced designers demonstrate more schema-driven than case-driven analogical reasoning, whilst novices show the reverse pattern of analogising (Ball et al., 2004) and can be distinguished by the way they decompose a problem (Ho, 2001). How novices and experienced designers design and shape products is still underexplored (Jagtag, 2018). Such single-domain studies based on architecture (Kavakli and Gero, 2002), engineering design (Cross and Clayburn Cross, 1998; Atman et al., 1999; Ahmed et al., 2003; Ball et al., 2004) and industrial design (Ho, 2001; Jagtag, 2018) provide insights on how novices and experienced professionals design within a domain.

However, the emergent conceptual move towards understanding the cognitive mechanisms of designing, through the use of methods from the neurosciences provide the means to investigate how designers change with experience and how this translates into neurophysiological activation. This opens the way to identify the drivers and triggers of change in design with implications for design education and design process modelling, in particular if correlated with protocol studies. Neurophysiological measurements offer a window to explore how these aspects unfold over time.

The research reported in this paper examines the neurophysiological activations throughout constrained and open design tasks performed by novice and experienced professionals from the domains of mechanical engineering and industrial design. We study how problem-solving and designing cognitive processes unfold and translate into neurophysiological activations. We describe preliminary results from a controlled experiment in which, by taking advantage of the temporal resolution of electroencephalography (EEG) and the use of low-cost portable equipment, significant differences in neurophysiological activations are found between problem-solving and design tasks for both cohorts. We report the comparison between domains elsewhere (Vieira et al., 2019b). In this paper we compare the neurophysiological activations between the novice and experienced designers across both domains. This is a convenience sample that can provide preliminary insights into how novices and experienced designers approach constrained problem-solving and open design tasks across the two domains. To avoid any blurring effect, we define novices as designers with less than 5 years of design experience and experienced professionals as designers with more than 5 years of experience.

\subsection{Electroencephalography studies of novice and experienced designers in design research}

Electroencephalographic activity reflects the summation of the synchronous activity of thousands or millions of pyramidal neurons that have similar spatial orientation. Their ions line up and create waves to be detected (Dickter and Kieffaber, 2014). EEG measures electromagnetic fields generated by this neural activity. Activity from deep sources of the brain is more difficult to detect than currents near the skull, thus EEG is more sensitive to cortical activity (Hinterberger et al., 2014, Dickter and Kieffaber, 2014). EEG offers high temporal resolution in the order of milliseconds in a portable device that makes it a suitable tool to investigate designing as a temporal activity.

Design studies based on EEG commenced more than 40 years ago (Martindale and Hines, 1975) investigating cortical activation during multiple tasks. Some 20 years later a study on design categorization tasks of experts and novices (Göker, 1997) showed that while novices try to solve assignments through deductive reasoning, experts prefer to apply their experience directly. The regions activated in the brain during these design problem-solving tasks vary according to the experience a test person. From this behavioral experiment novices show a longer activity in the frontal regions whereas the experts show longer activity in the parietal regions of the brain.

Current results from controlled experiments based on art categorization tasks and single-domain expert designers focus on visual attention and association (Liang et al., 2017) though no comparison with novices is provided. In the last 10 years, single domain-related EEG design studies have been used to understand the acts of designing from a neurophysiological perspective (Liu et al., 2018; Vieira et al., 2019a). Multiple domain experiments show preliminary comparison results (Vieira et al., 2019b).

\subsection{Problem-solving and designing in constrained and open design spaces}

The concepts of problem space and solution space have populated design research (Dorst and Cross, 2001; Kruger and Cross, 2006) throughout the more than half a century of formal study of design (Jones and Thornley, 1963). One of the core research questions is whether designing, as a cognitive process, is 
distinct from problem-solving (Goel and Pirolli, 1992, 1989; Visser, 2009). In problem-solving all the characteristics of the problem are firstly defined, therefore problem-solving is a closed task based on known constants and known variables. Designing relates to the search for variables that are initially not known, such variables are context sensitive, can bring change and have variant meanings according to the design situation (Gero, 1990). In designing the characteristics of the problem are not all defined from the start making designing an open task. Distinguishing novices and experienced designers' neurophysiological activations while designing and problem-solving has implications for design research and design education in particular. The study is based on the analysis of mechanical engineers' and industrial designers' neurophysiological activations using an EEG headset in the context of performing problem-solving and design tasks in a laboratory setting. The aims of the present study are to:

- investigate the neurophysiological activation differences of novice and experienced professionals from the domains of mechanical engineering and industrial design when designing and problemsolving;

In the research reported in this paper we test the following hypotheses:

Hypothesis 1: the neurophysiological activations of novice professional designers when problemsolving and designing are significantly different.

Hypothesis 2: the neurophysiological activations of experienced professional designers when problemsolving and designing are significantly different.

Hypothesis 3: the neurophysiological temporal distributions of activations of problem-solving and designing are significantly different for novice professional designers.

Hypothesis 4: the neurophysiological temporal distributions of activations of problem-solving and designing are significantly different for experienced professional designers.

\section{Methods}

The study focuses on a subset of 55 experiment sessions of mechanical engineering and industrial design professionals. The tasks and experimental procedure were piloted prior to the full study. The hypotheses are tested by comparing the neurophysiological activations during the design tasks with those of the problem-solving task. We compare absolute values known as transformed power (Pow), defined in 2.5, and use the problem-solving EEG neurophysiological activation as the base for comparison.

\subsection{Participants}

Participants were based on a convenience sample, the subset of 55 experimental sessions comprises 26 mechanical engineers and 29 industrial designers. Final results are based on a subset of 36 right-handed participants, 18 mechanical engineers, aged 25-40 $(\mathrm{M}=28.9, \mathrm{SD}=4.2), 10$ men (age $\mathrm{M}=29.0, \mathrm{SD}=5.3$ ) and 8 women (age $\mathrm{M}=28.7, \mathrm{SD}=2.5$ ); and 18 industrial designers, aged 25-43 $(\mathrm{M}=31.7, \mathrm{SD}=7.3), 10$ men (age $\mathrm{M}=35.1, \mathrm{SD}=7.2$ ) and 8 women (age $\mathrm{M}=27.5, \mathrm{SD}=5.1$ ). The participants are all professionals (experience $\mathrm{M}=5.9, \mathrm{SD}=6.0$ ). The sample has 20 participants with experience up to 5 years, and 16 participants with experience above 5 years. An independent-samples t-test was conducted to compare participants with up to 5 years of experience with those with more than 5 years of experience from the cohorts of mechanical engineers and industrial designers. There was no significant difference between experienced professionals, mechanical engineers $(\mathrm{M}=9.5, \mathrm{SD}=3.5, \mathrm{~N}=8)$ and experienced industrial designers $(\mathrm{M}=12.3, \mathrm{SD}=4.5, \mathrm{~N}=8), \mathrm{t}=1.22 p=0.24$. An independent-samples $\mathrm{t}$-test was conducted to compare participants with up to 5 years of experience from the cohorts of mechanical engineers and industrial designers. There was no significant difference between novice professionals, mechanical engineers $(\mathrm{M}=3.0$, $\mathrm{SD}=1.4, \mathrm{~N}=10)$ and novice professional industrial designers $(\mathrm{M}=2.5, \mathrm{SD}=0.7, \mathrm{~N}=10), \mathrm{t}=0.87 p=0.39$. Thus, we compared the transformed power (Pow) of novices and experienced designers across tasks and domains. This study was approved by the local ethics committee of the University of Porto.

\subsection{Experiment design}

We adopted the problem-solving and layout design tasks described in the Alexiou et al. (2009) fMRIbased study. We matched Tasks 1 and 2 with the problem-solving and design tasks (Alexiou et al., 2009) 
in terms of requests, number of constraints, stimuli and number of instructions. Task 1 is considered a problem-solving task as the problem itself is well-defined, and the set of equivalent solutions is unique (Alexiou et al., 2009). We extended that experiment by adding an open layout design task to produce a block experiment in order to determine whether the open layout design task produces different results to the semi-closed layout Task 2. Both design tasks have no predetermined final state and the tasks are openended. The block experiment consisted of a sequence of 3 tasks: problem-solving, basic layout design, and open layout design, Figure 1. We added a fourth open design task that uses free-hand sketching after Task 3. Task 4 is an ill-defined and fully unconstrained task unrelated to formal problem-solving. Each participant was given two sheets of paper (A3 size) and three instruments, a pencil, graphite and a pen. The Tasks 2, 3 and 4 require defining the design space in a successive order of complexity, Table 1 . The set of four tasks was preceded by a pre-task. Detailed description can be found in Vieira et al. (2019b). As with all block experiments, each subsequent task was potentially primed by the previous task. The Mikado pickup-sticks game was given to the participants to play in the breaks between tasks.

Table 1. Description of the problem-solving, basic design and open design tasks

\begin{tabular}{l|l|l|l}
\hline \multicolumn{1}{c|}{$\begin{array}{c}\text { Task 1 } \\
\text { Problem-solving }\end{array}$} & \multicolumn{1}{c|}{$\begin{array}{c}\text { Task 2 } \\
\text { Basic layout design }\end{array}$} & \multicolumn{1}{c}{$\begin{array}{c}\text { Task 3 } \\
\text { Open layout design }\end{array}$} & \multicolumn{1}{c}{$\begin{array}{c}\text { Task 4 } \\
\text { Open sketching design }\end{array}$} \\
\hline $\begin{array}{l}\text { In Task 1 the design } \\
\text { of a set of furniture is } \\
\text { available and three } \\
\text { conditions are given } \\
\text { as requirements. The } \\
\text { task consists of }\end{array}$ & $\begin{array}{l}\text { In Task 2 the same design } \\
\text { set of furniture is available } \\
\text { and three requests are } \\
\text { made. The basic design } \\
\text { task consists of placing the magnetic } \\
\text { pieces inside a given } \\
\text { area of a room with a } \\
\text { door, a window and a } \\
\text { balcony. }\end{array}$ & $\begin{array}{l}\text { furniture inside a given } \\
\text { room area according to } \\
\text { each participant notions of } \\
\text { available is complemented } \\
\text { with a second board of } \\
\text { movable pieces that } \\
\text { comprise all the fixed } \\
\text { elements of the previous } \\
\text { tasks, namely, the walls, } \\
\text { the door, the window and } \\
\text { the balcony. The }\end{array}$ & $\begin{array}{l}\text { In the free-hand } \\
\text { Task 4, the participants } \\
\text { are asked to: propose and } \\
\text { represent an outline design } \\
\text { for a future personal } \\
\text { entertainment system }\end{array}$ \\
\hline
\end{tabular}

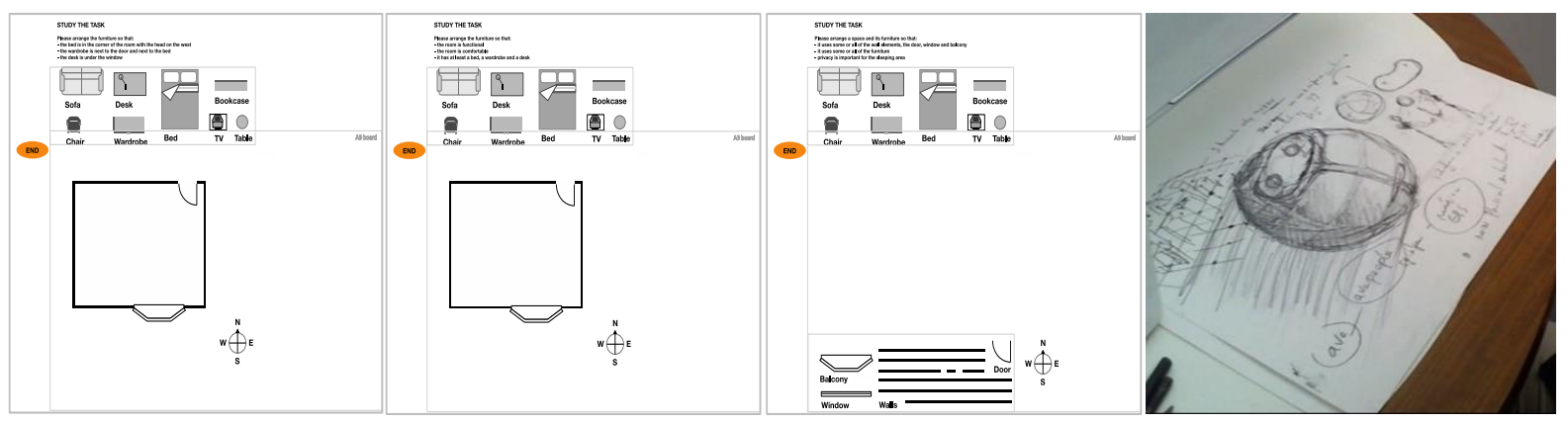

Figure 1. Depiction of the problem-solving Task 1, layout design Task 2, open layout design Task 3 and open free-hand sketching design Task 4

\subsection{Setup and procedure}

A tangible interface for individual task performance was built based on magnetic material for easy handling, Figure 2. Differently from the original tasks (Alexiou et al., 2009), the magnetic pieces were placed at the top of the vertical magnetic board to prevent signal noise due to eye and head horizontal movements. One researcher was present in each experiment session to instruct the participant and to check for recording issues. A period of 10 minutes for setting up and a few minutes for a short introduction are necessary for informing each participant, reading and signing of the consent agreement and set the room temperature. The researcher checked metallic accessories for electromagnetic interference and contact lenses. The researcher sat each participant at the desk and checked their posture. A more detailed procedure is described in Vieira et al. (2019b). The researcher followed a script to conduct the experiment so that each participant was presented with the same information and stimuli. Before each 
task, participants were asked to start by reading the text. The participants were asked to stay silent during the tasks and use the breaks for talking and clarifying doubts. If needed, extra time was given to the participants, in particular in Tasks 3 and 4, so they could find a satisfactory solution.

\subsection{Data collection methods}

EEG activity was recorded using a portable 14-channel system Emotiv Epoc+. Electrodes are arranged according to the 10-10 I.S standard configuration, Figure 2. Electromagnetic interference of the room was checked for frequencies below $60 \mathrm{~Hz}$. The participants performed the tasks using the physical magnetic board, with two video cameras capturing the participant's face and activity and an audio recorder, Figure 2. All the data captures were streamed using Panopto software (https://www.panopto.com/), Figure 2. The mechanical engineers and industrial designers used in this study came from a total of 100 experimental sessions of architects, mechanical engineers, industrial designers and graphic designers, which constitute our major data set. Of the 100 sessions 90 took place at the University of Porto. Ten sessions took place in the Design Hub of Mouraria, Lisbon, in rooms with the necessary conditions for the experiment, such as natural lighting sufficient for performing experiments between 9:00 and 15:00 and no electromagnetic interference.

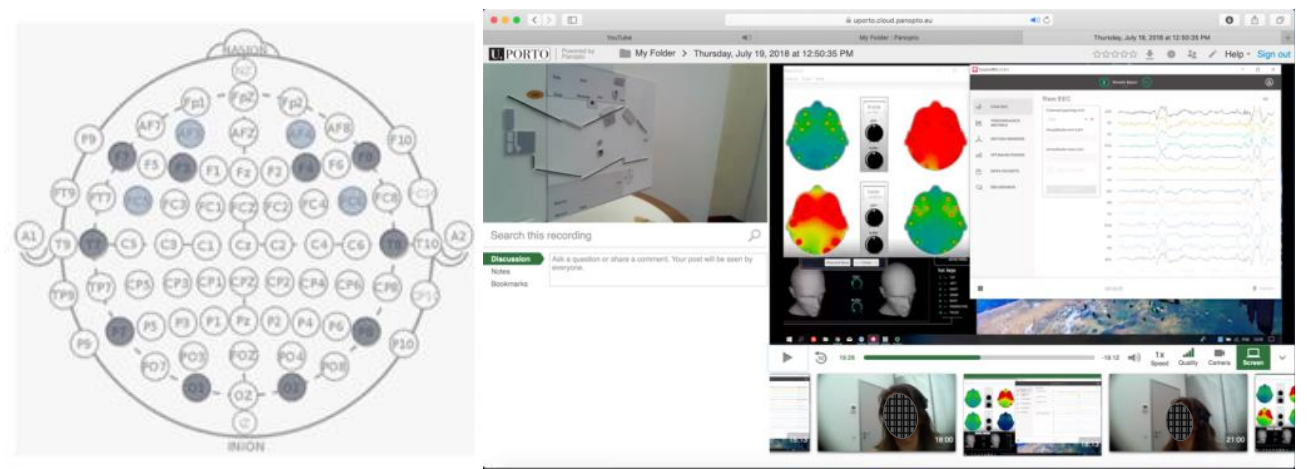

Figure 2. Emotiv Epoc+ electrodes arrangement (10-10 I.S.) and screen capture depicting audio, video and screen

\subsection{Data processing methods}

Due to left-handedness or EEG or video recording issues seven experiments were excluded, leaving 48 sessions. The fourteen electrodes collected data with a $256 \mathrm{~Hz}$ sampling rate, low cutoff $0.1 \mathrm{~Hz}$, high cutoff $50 \mathrm{~Hz}$. We adopted the blind source separation (BSS) technique based on canonical correlation analysis (CCA) for the removal of muscle artifacts from EEG recordings (De Clercq et al., 2006) adapted to remove the short EMG bursts due to articulation of spoken language (Vos et al., 2010). A detailed description is provided in Vieira et al. (2019a). Data analysis included total power values on individual and aggregate levels using MatLab and EEGLab open-source software. All the EEG segments of the recorded data were used for averaging throughout the entire tasks, from beginning to end. All the tasks of this experiment involve thinking and motion. The motor actions involved in the tasks using the tangible interface versus the free-hand sketching and their corresponding EEG signals are of the same source, thus we claim that the BSSCCA procedure filters $90 \%$ of the signal from artifacts. We report results on transformed power (Pow), the mean of the squared values of microvolts per second $\left(\mu \mathrm{V}^{2}\right)$ for each electrode processed signal per task. This measure tells us about the amplitude of the signal per channel and per participant magnified to squared values. We present Pow values of aggregates of participants' individual results, for each cohort, per total task and for each task deciles for the temporal analysis. After determining the outliers as described below, we calculated the mean and standard deviation of each measure for each cohort. A z-transform was conducted in the analysis of Pow across tasks for each of the participants' data to determine outliers; the criteria for excluding participants were based on the evidence of 6 or more threshold z-score values above 1.96 or below -1.96 and individual measurements above 2.81 or below -2.81 . This resulted in a further 8 sessions being excluded leaving 40. After the division of the Pow into time deciles amplitude leading to two and a half standard deviations from the mean as threshold values were excluded per channel, 4 experiments were further excluded leaving 36 , coincidentally 18 per domain. 


\subsection{Data analysis methods}

We carried out statistical analyses of total and temporal neurophysiological activations based on dividing the design sessions into time deciles. We performed standard analyses based on the design of the experiment: a repeated-measures ANOVA with pairwise comparisons to follow up on specific differences with task, hemisphere, electrode and decile as within-subject factors for each cohort. These analyses were performed for the dependent variable of Pow and for all the within-subject variables. The threshold for significance in all the analyses is $p \leq .05$.

\section{Analysis of results}

From the analysis of the transformed power (Pow) results across the 36 participants indicate that the tasks can be distinguished from each other for the novices and the experienced designers.

\subsection{Problem-solving and design tasks of novice and experienced professionals}

To compare the Pow of all the tasks we first performed an analysis by running a $5 \times 2 \times 7$ repeatedmeasurement ANOVA, with the within-subject factors of task, hemisphere and electrode. From the analysis of the 20 novice participants, we found significant main effects, Table 2.

Table 2. Significant effects from the ANOVA based on the $\mathbf{2 0}$ novice designers

\begin{tabular}{l} 
Significant main effects \\
\hline task $(p<.01)$; hemisphere $(p<.001)$; electrode $(p=.02)$ \\
\hline
\end{tabular}

In addition, we conducted pairwise comparisons for hemisphere, electrode and task. The pairwise comparisons revealed that the open design sketching Task 4 differs significantly from:

- $\quad$ Problem-solving Task $1(p=.01)$

- Layout design Task $2(p=.01)$ and open layout design Task $3(p=.02)$

The pairwise comparisons further revealed differences between hemispheres for the 7 electrodes and tasks. In Figure 3, left image, we report on significant $(p \leq .05)$ pairwise comparisons found between Task 1 (problem-solving) and Task 4 (open free-hand sketching design).

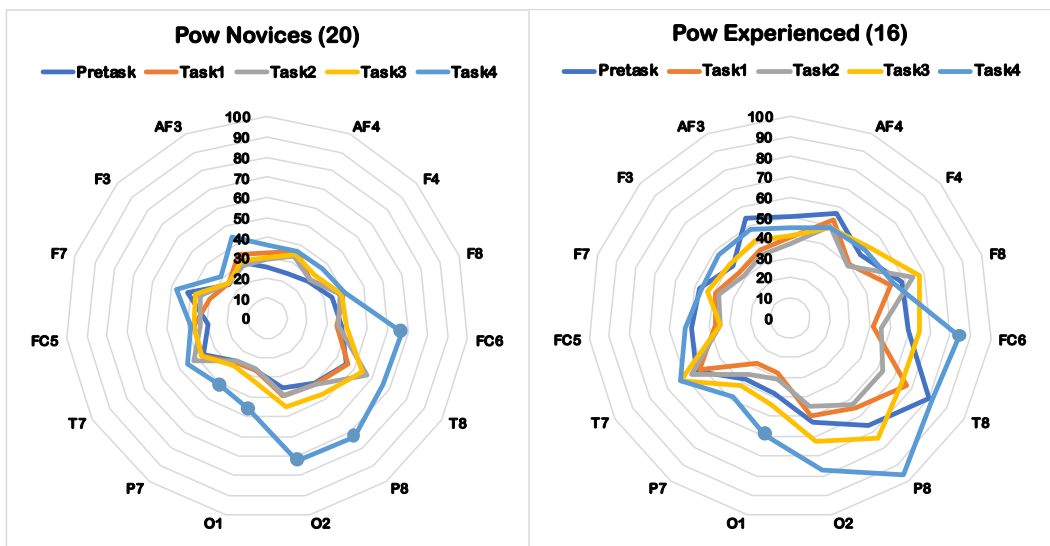

Figure 3. Transformed power (Pow) of the five tasks for novice and experienced designers, labels refer to channels in standard locations (significant task differences per electrode are shown as solid circles)

The radar plot simulates the two hemispheres by distributing the electrodes (10-10 IS) symmetrically around a vertical axis. Total Pow scores per electrode can be considered by comparing with the vertical scale and across the different tasks. Circles represent the channels with activation of statistically significant differences in Task 4 from Task 1, namely, FC6 ( $p<.01)$; P8 ( $p=.02)$; O2 ( $p<.01)$ in the right hemisphere, and P7 ( $p=.04)$ and $\mathrm{O} 1(p=.01)$ in the left hemisphere. Task 4 shows higher activations across channels. From the analysis of the 16 experienced designers we found significant main effects as depicted in Table 3 . 
Table 3. Significant effects from the ANOVA based on the 16 experienced designers

\begin{tabular}{l}
\hline Significant main effects \\
\hline hemisphere $(p<.001) ;$ electrode $(p<.01)$ \\
\hline
\end{tabular}

The pairwise comparisons revealed that the open design sketching Task 4 and open layout design Task 3 respectively differ significantly from:

- Problem-solving Task $1(p=.02)$

- Layout design Task $2(p<.01)$

The pairwise comparisons further revealed differences between hemisphere for the 7 electrodes and tasks. In Figure 3, image on the right, we report on significant $(p \leq .05)$ pairwise comparisons found between Task 1 (problem-solving) and Task 4 (open free-hand sketching design) of experienced designers. Circles represent the channels with activation of statistically significant differences in Task 4 from Task 1, namely, FC6 $(p<.01)$ in the right hemisphere, and O1 $(p<.01)$ in the left hemisphere. EEG neurophysiological activations are associated with conceptual expansion (Abrahams, 2019). Novice designers show higher activation in channels (F7, AF3), associated with deductive reasoning and channels of the right occipitotemporal cortex. Experienced designers show even higher activation in channels associated with inductive reasoning (F3, P7), channels of the right dorsolateral prefrontal cortex (AF4, F8, FC6, ) and right and left occipitotemporal cortices (T8, P8, O2, O1, P7 and T7).

\subsection{Analysis of temporal stages of novice and experienced professionals}

Designing is a temporal activity, segmenting across time shows how designing unfolds in stages. Different cognitive behaviours can be found when design sessions are divided into deciles (Kan and

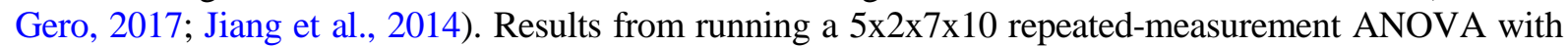
the within-subject factors of task, hemisphere, electrode and decile, for each cohort are presented below. From the analysis of the 20 novice professional designers, we found significant main effects, Table 4. General higher activations of channels of the right occipitotemporal cortex and left secondary visual cortex are shown in the open space sketching design Task 4 across deciles, Figure 4.

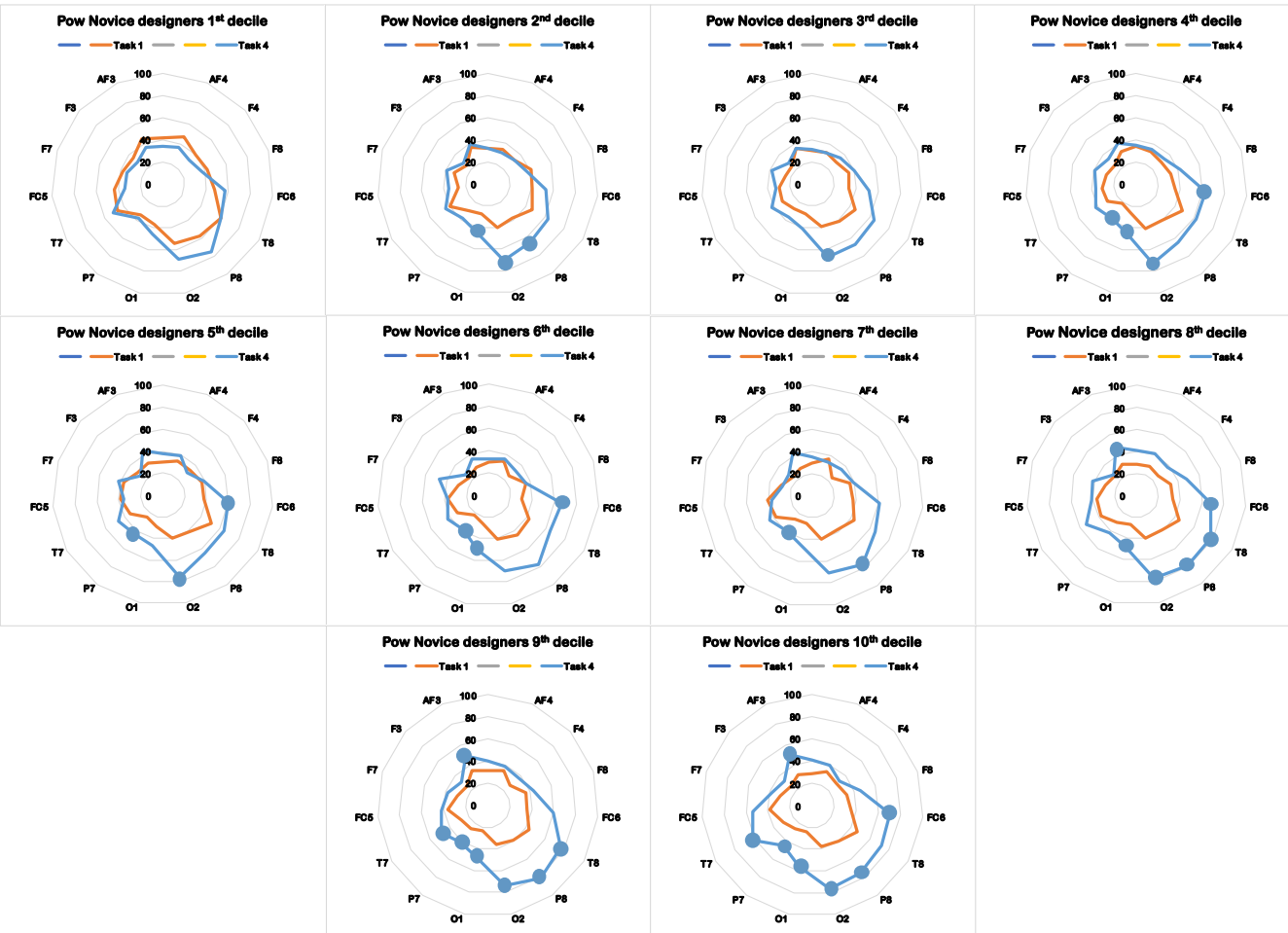

Figure 4. Channels of significant differences for novice designers between Task 1 and Task 4 , shown as solid circles 
Statistically significant differences between the open design Task 4 and constrained problem-solving Task 1 occur in the left prefrontal cortex, in the left and right occipitotemporal cortices. Decreased activation from Task 4 to Task 1 happens in some channels of the prefrontal cortex.

Table 4. Significant effects from the ANOVA based on the 20 novice designers

\begin{tabular}{l} 
Significant main effects \\
\hline task $(p<.01) ;$ hemisphere $(p<.01)$; electrode $(p<.001)$ \\
\hline
\end{tabular}

The pairwise comparisons revealed that the open design sketching Task 4 differs significantly from:

- Problem-solving Task $1(p<.01)$

- Basic layout design Task 2(p<.01) and Open layout design Task $3(\mathrm{p}<.01)$

From the analysis of the 16 experienced professional designers, significant main effects, Table 5 . General higher activations of the channels of the right occipitotemporal cortex and secondary visual cortex are shown in Task 4 across deciles, Figure 5.

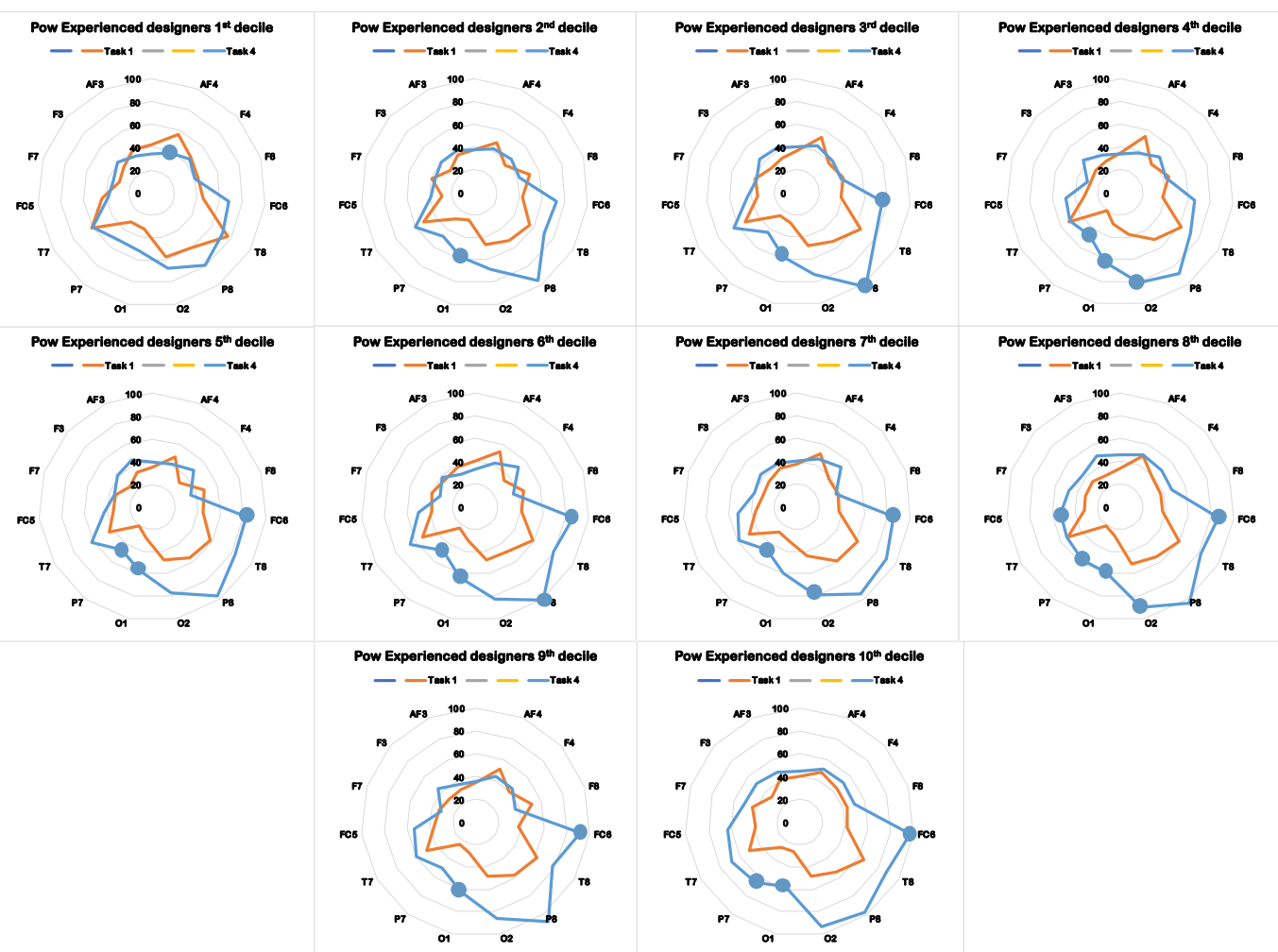

Figure 5. Channels of significant differences for experienced designers from Task 1 to Task 4 , shown as solid circles

Statistically significant differences between problem-solving Task 1 and Task 4 occur in the temporal and secondary visual cortices. The channels with decreased activation from Task 1 to Task 4 are located in the prefrontal cortex as well.

Table 5. Significant effects from the ANOVA based on the 16 experienced participants

\begin{tabular}{l} 
Significant main effects \\
\hline task $(p=.04)$; hemisphere $(p<.001)$; electrode $(p=.04)$ \\
\hline
\end{tabular}

The pairwise comparisons revealed that the open design sketching Task 4 and open layout design Task 3 respectively differ significantly from:

- Problem-solving Task $1(p<.05)$ and basic layout design Task $2(p=.04)$

- Basic layout design Task $2(p=.03)$ 


\section{Conclusion}

Results from this analysis of the EEG data of the 36 participants show significant differences of neurophysiological activations between problem-solving Task 1 and the open design sketching Task 4 for both novice and experienced professionals across the two cohorts of mechanical engineers and industrial designers. Thus, preliminary evidence provides initial support for hypotheses 1 and 2: the neurophysiological activations of experienced and novice professional designers when problemsolving and designing are significantly different. Experienced professionals show higher transformed power (Pow) across all channels than do novice designers.

The temporal distributions of neurophysiological activations of novice and experienced professionals also show significant differences between problem-solving Task 1 and the open design sketching Task 4 providing initial support for hypothesis 3 and 4: the neurophysiological temporal distributions of activations of problem-solving and designing are significantly different for experienced and novice professional designers. Novice designers show higher variation of Pow between Tasks 1 and 4 . Further analysis will focus in the following areas: (i) differences between novice and experienced professionals, (ii) connecting brain region activations to cognitive functions associated with those regions, (iii) band filtering, and (iv) a more fine grained analysis addressing temporal changes.

These results show the effects of experience in the neurophysiological activations when tackling different problem statements. Studying the brains of designers is relatively new and provides a more objective measurement of designing through neurocognition than cognitive studies alone. Such studies have the potential to be able to answer research questions that are currently difficult to answer. Questions such as: how persistent are effects of educational interventions? Further advancements can enable novice designers to better monitor their thinking modes during the design process and therefore improve design performance and provide knowledge on how change can be predicted in design. Novel experimental techniques are under development to allow for a direct correlation between design cognition, obtained through protocol analysis, with these objective neurophysiological measurements.

\section{Acknowledgements}

This research was supported by the Unity of Conceptual and Experimental Validation of INEGI-Feup. The research is funded by the Portuguese Foundation for Science and Technology, grant number SFRH/BPD/104281/2014. The second author acknowledges the support of the US National Science Foundation, Grant No. CMMI-1762415 and EEC-1929896. Any opinions, findings, and conclusions or recommendations expressed in this material are those of the authors and do not necessarily reflect the views of the National Science Foundation.

\section{References}

Abrahams, A. (2019), The neuroscience of creativity. Cambridge University Press. https://doi.org/10.1017/9 781316816981

Alexiou, K. et al. (2009), "Exploring the neurological basis of design cognition using brain imaging: some preliminary results", Design Studies, Vol. 30 No. 6, pp. 623-647. https://doi.org/10.1016/j.destud.2009.05.002

Ahmed, S., Wallace, K.M. and Blessing, L.T.M. (2003), "Understanding the differences between how novice and experienced designers approach design tasks", Research in Engineering Design, Vol. 14 No. 1, pp. 111. https://doi.org/10.1007/s00163-002-0023-z

Atman, C.J. et al. (1999), “A comparison of freshman and senior engineering design processes", Design Studies, Vol. 20 No. 2, pp. 131-152. https://doi.org/10.1002/j.2168-9830.2007.tb00945.x

Ball, L.J., Ormerod, T.C. and Morley, N.J. (2004), "Spontaneous analogising in engineering design: a comparative analysis of experts and novices", Design Studies, Vol. 25 No. 5, pp. 495-508. https://doi.org/ 10.1016/j.destud.2004.05.004

Crutcher, R.J. (1994), "Telling what we know: The use of verbal report methodologies in psychological research", Psych. Sci. Vol. 5, pp. 241-244. https://doi.org/10.1111/j.1467-9280.1994.tb00619.x

Cross, N. and Clayburn Cross, A. (1998), "Expertise in Engineering Design", Research in Engineering Design, Vol. 10 No. 3, pp. 141-149. https://doi.org/1007/BF01607156

De Clercq, W. et al. (2006), "Canonical correlation analysis applied to remove muscle artifacts from the electroencephalogram", IEEE Transactions on Biomedical Engineering, Vol. 53, pp. 2583-2587. https://doi.org/10.1109/TBME.2006.879459

Dickter, C. and Kieffaber, P. (2014), EEG Methods for the Psychological Sciences. Sage, https://doi.org/10.4 $135 / 9781446270356$ 
Dorst, K. and Cross, N. (2001), "Creativity in the design process: Co-evolution of problem-solution", Design Studies Vol. 22, pp. 425-437. https://doi.org/10.1016/S0142-694X(01)00009-6

Dreyfus, H., and Dreyfus, S. (1988), Mind over Machine. Free Press.

Ericsson, K. and Simon, H. (1993), Protocol Analysis; Verbal Reports as Data, MIT Press.

Gero, J. S. (1990), "Design Prototypes: a knowledge representation schema for design”, AI Magazine, Vol. 11 No. 4, pp. 26-36. https://doi.org/10.1609/aimag.v11i4.854

Goel, V. and Pirolli, P. (1989), "Motivating the Notion of Generic Design within Information Processing Theory: The Design Problem Space", AI Magazine Vol. 10 No. 1, pp. 18-36. https://doi.org/10.1609/aim ag.v10i1.726

Goel, V. and Pirolli, P. (1992), “The structure of design problem spaces”, Cognitive Science, Vol. 16, pp.395-429. https://doi.org/10.1207/s15516709cog1603_3

Göker, M. (1997), “The effects of experience during design problem solving”, Design Studies, Vol 18, pp.405-426. https://doi.org/10.1016/S0142-694X(97)00009-4

Hinterberger, T., Zlabinger, M. and Blaser, K. (2014), "Neurophysiological correlates of various mental perspectives", Frontiers in Human Neuroscience, Vol 8, pp. 1-16. https://doi.org/10.3389/fnhum.2014.00637

Ho, C.H. (2001), "Some phenomena of problem decomposition strategy for design thinking: differences between novices and experts", Design Studies, Vol. 22 No. 1, pp. 27-45. https://doi.org/10.1016/S0142-694X(99)00030-7

Jiang, J., Gero, J.S., Yen, C. (2014), "Exploring designing styles using Problem-Solution indexes", In Gero, J.S. (ed), Design Computing and Cognition'12, Springer, pp. 85-101. https://doi.org/10.1007/978-94-017-9112-0

Jones, J. C. and Thornley, D. G. (eds). (1963), Conference on Design Methods. Oxford, UK: Pergamon Press.

Jagtap, S. (2018), "Shaping products: Differences between expert and novice industrial designers", 15th International Design Conference. https://doi.org/10.21278/idc.2018.0159.

Kan, J. W. T. and Gero, J. S. (2017), Quantitative Methods for Studying Design Protocols. Springer. https://doi.org/10.1007/978-94-024-0984-0

Kavakli, M. and Gero, J. (2002), "The Structure of Concurrent Cognitive Actions: a case study on novice and expert designers", Design Studies, Vol. 23 No. 1, pp. 25-40. https://doi.org/10.1016/S0142-694X(01)00021-7

Kruger, C. and Cross, N. (2006), "Solution driven versus problem driven design: Strategies and outcomes", Design Studies, Vol. 27 No. 5, pp.527-548. https://doi.org/10.1016/j.destud.2006.01.001

Liang, C. et al. (2017), "Visual attention and association: An electroencephalography study in expert designers", Design Studies Vol. 48, pp. 76-95. https://doi.org/10.1016/j.destud.2016.11.002

Liu, L. et al. (2018), "An EEG study of the relationship between design problem statements and cognitive behaviors during conceptual design", Artificial Intelligence for Engineering Design, Analysis and Manufacturing Vol. 32, pp. 351-362. https://doi.org/10.1017/S0890060417000683

Martindale, C. and Hines, D. (1975), "Creativity and Cortical activation during Creative, Intellectual and EEG Feedback Tasks", Biological Psychology, Vol. 3, pp. 91-100. https://doi.org/10.1016/0301-0511(75)90011-3

Vieira, S. et al. (2019a), "Understanding the design neurocognition of mechanical engineers when designing and problem-solving", ASME, https://doi.org/10.1115/DETC2019-97838

Vieira, S. et al. (2019b), "Comparing the Design Neurocognition of Mechanical Engineers and Architects: A Study of the Effect of Designer's Domain". in Proceedings of the 22nd International Conference on Engineering Design (ICED19), Delft, The Netherlands, 5-8 August, p. 1853-1862. https://doi.org/10.1017/dsi.2019.191

Visser, W. (2009), "Design: one, but in different forms", Design Studies Vol. 30 No. 3, pp. 187-223. https://doi.org/10.1016/j.destud.2008.11.004

Vos, D. et al. (2010), "Removal of muscle artifacts from EEG recordings of spoken language production", Neuroinform, Vol. 8, pp. 135-150. https://doi.org/10.1007/s12021-010-9071-0. 